\title{
Cooling Hadron Beams with Electron Pulses
}

\author{
Pulsed electron beams can be used to cool beams of ions and protons \\ circulating in a hadron storage ring-a promising development for future \\ high-energy accelerators.
}

\author{
By Erika K. Carlson
} $\mathrm{n}$ hadron accelerators, the accelerated ion or proton beam heats up over time, degrading in quality. A popular method for counteracting beam degradation involves using an electron beam to cool the ions: the electron beam mixes with the hadron beam and cools it down as the two beams travel along the same path. Now, Max Bruker of Thomas Jefferson National Accelerator Facility in Virginia and colleagues have demonstrated an electron cooling technique that works with electron pulses, rather than with continuous electron beams [1]. The bunched scheme will be applicable to future hadron accelerators that are expected to achieve higher particle energies than existing facilities.

Existing techniques for cooling with electrons accelerate direct current ( $\mathrm{dc}$ ) electron beams using static high voltages. But dc acceleration only works up to a certain energy; to produce electron cooling beams at higher energies, researchers would need to accelerate the electrons with radio-frequency pulses.

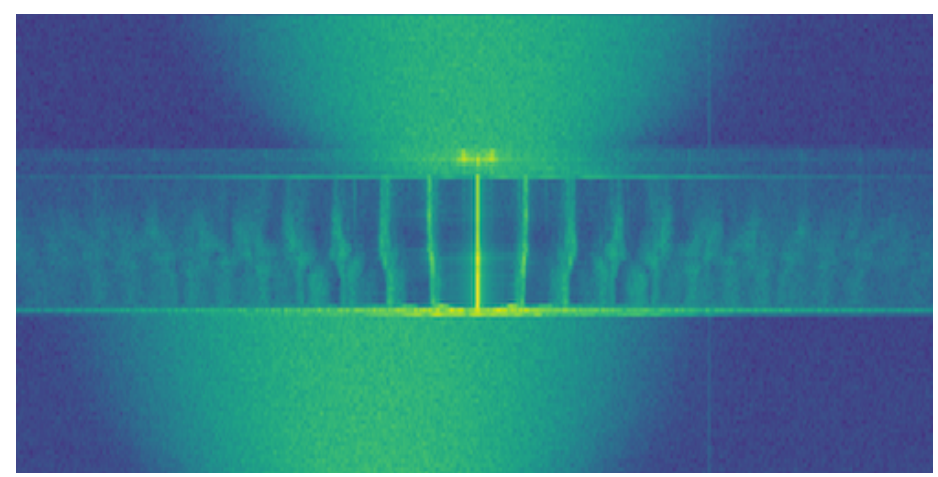

Credit: M. Bruker et al. [1]
This approach produces electron bunches, rather than a continuous beam. To understand how the time structure of such an electron beam might affect its ability to cool a hadron beam, Bruker and colleagues performed experiments at an ion ring facility. The researchers modified a traditional dc electron cooler so that it delivered a pulsed beam. They found that the bunched electron beam could efficiently cool the hadron beam, provided that the timings and lengths of the electron bunches were properly controlled to match those of the hadron beam. As high-energy facilities such as the Electron-Ion Collider are being designed, the new scheme provides a promising cooling option, the team says.

Erika K. Carlson is a Corresponding Editor for Physics based in New York City.

\section{REFERENCES}

1. M. Bruker et al., "Demonstration of electron cooling using a pulsed beam from an electrostatic electron cooler," Phys. Rev. Accel. Beams 24, 012801 (2020). 